\title{
Characteristics of Facial Skin and Demodex Mites
}

\author{
Kubanov Alexey ${ }^{1}$, GallyamovaYulia ${ }^{2}$, KravchenkoAnzhela ${ }^{3 *}$ \\ ${ }^{1}$ Head of department of dermatovenereology and cosmetology of Russian medical academy of continuous \\ professional education of the Ministry of Healthcare of the Russian Federation, Russia \\ ${ }^{2}$ Professor of department of dermatovenereology and cosmetology of Russian medical academy of continuous \\ professional education of the Ministry of Healthcare of the Russian Federation, Russia \\ ${ }^{3}$ Russian medical academy of continuous professional education of the Ministry of Healthcare of the Russian \\ Federation, Russia
}

*Corresponding Author: KravchenkoAnzhela, Russian medical academy of continuous professional education of the Ministry of Healthcare of the Russian Federation, Russia, Email: angkravchenko@ gmail.com

Abstract: The main aim of the study is to evaluate a connection between morphofunctional characteristics of the skin of patients with rosacea and presence of Demodex mites.

Materials and Methods: 180 people, including patients with rosacea with presence and absence of Demodex mites and healthy volunteers, were examined using digital video camera "Aramo SG".

Results: The statistic differences was find between patients of all groups in terms of parameters of moisture, fat content, pigmentation, elasticity, smoothness, pore size.

Conclusion: Morphofunctional characteristics of facial skin become worse with a presence of Demnodex mites in patiens with rosacea.

Keywords: rosaces, Demodex mites, morphofunctional characteristics, skin types.

\section{INTRODUCTION}

Rosacea, being one of the most spread out diseases, remains an urgent problem in dermato venereo logy. Among the various causes of the appearance of rosacea, a parasitic theory plays a role [1]. According to her, the formation of papules and pustules on the face skin is promoted by the Demodex mite.

The main nutrient material for Demodex mites is the secretion of sebaceous glands, as well as skin scales [2, 3, 4]. To date, until the end it is not known what changes in the morphofunctional characteristics of the skin contribute to parasitizing Demodex mites.

The aim of the study was to establish a relationship between the change in the morphofunctional characteristics of facial skin in rosacea patients and infection with Demodex mites.

\section{Materials And Methods}

The study was conducted in compliance with the provisions of the Helsinki Declaration (1964) on Medical Ethics. The study protocol was approved at the meeting of the Ethical Committee of the Russian Medical Academy of
Continuous Professional Education. All patients who participated in the study signed the "Informed consent of the participant in biomedical research".

During the study, a total of 180 people (men and women) were examined. The study included healthy individuals, patients diagnosed with rosacea, with the presence and absence of Demodex mites. The diagnosis of "Rosacea" was established based on the clinical picture of the disease.

Clinical and morphofunctional assessment of the skin condition of men and women over 18 years of age was carried out on a consultative and diagnostic method. During the study, three groups were formed: two groups of patients (main and control) and a comparison group consisting of healthy individuals:

I group (main) - rosacea patients with the presence of Demodex mites $(n=60)$,

II group (control group) - patients diagnosed with rosacea with no Demodex mites $(n=60)$,

III (the group of comparison) - healthy persons are included $(n=60)$. 
Demodex mites were identified by standard method of light microscopy of skin scrapers. Enlargement of miscroscope was $\times 10$ and $\times 40$.
Figure 1 demonstrates a Demodex mite by microscopy of skin scrapers.

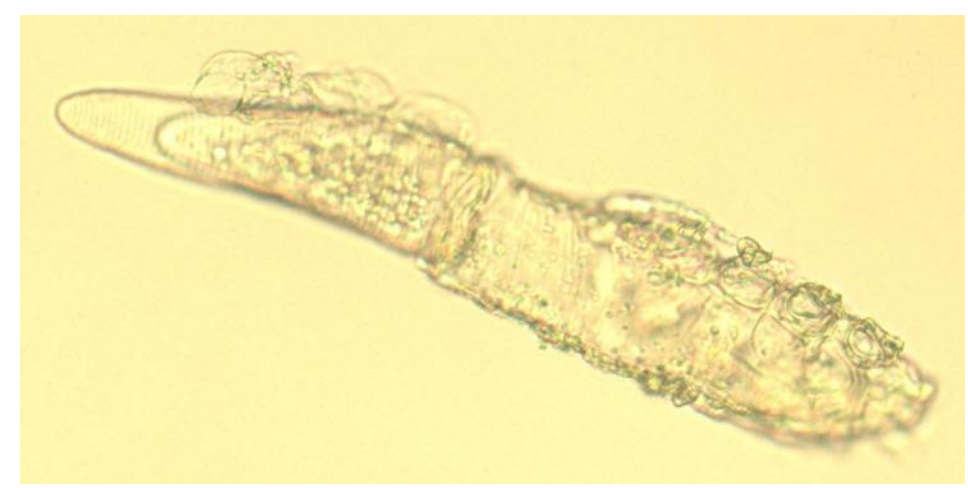

Fig1. Identification of Demodex mites by light microscopy of skin scrapers

To determine the morphofunctional characteristics of the facial skin in patients with rosacea I and II groups, as well as healthy individuals of group III, a dermatoscopy was carried out on a digital video camera "Aramo SG" using Skin XP Pro 2.0.

All the subjects studied the following morphofunctional characteristics of the skin: moisture; fat content; elasticity; smoothness (skin relief); pigmentation; pore size. With the help of litmus test strips, the $\mathrm{pH}$ of the facial skin of the respondents was measured.

The statistical processing of data was carried out by the packages of SPSS 21 . The interrelation of categorical indicators was established by the exact method of Fisher. To assess the significance of the difference in the size of the follicles, one-way analysis of variance was used with paired comparisons. To assess the significance of the differences in the absence of a normal distribution, the Mann-Whitney criteria, the Kruskal-Wallis multiple comparison criteria, were used. Differences were considered significant at $\mathrm{p}<0.05$.

\section{RESUltS}

Posacea patients complicated and un complicated by Demodex mites represents in Figures 2 and 3.

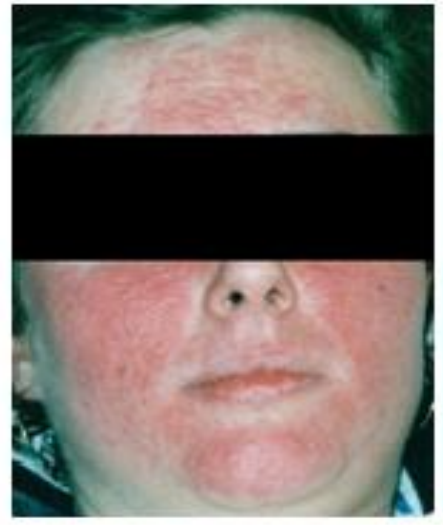

Fig2. Patient, 32 years old, Igroup

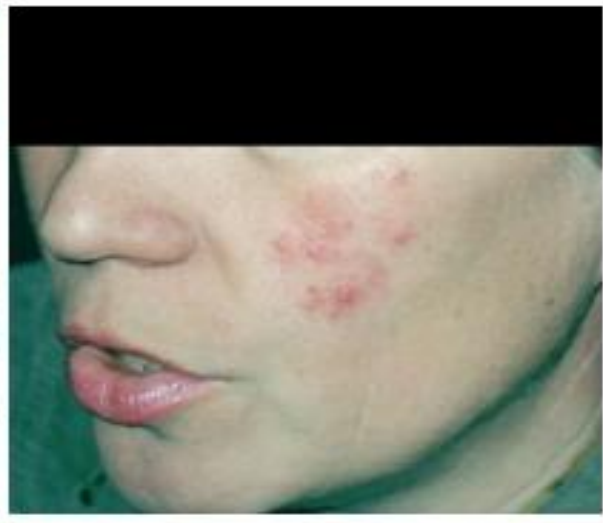

Fig3. Patient, 54 years old, IIgroup

Diagnosis: Rosacea, papulopustules form

well as comparative data between the groups, are presented in Table 1.
Diagnosis: Rosacea, papulopustules form, Complicated by Demodex mites

The data obtained for the morphofunctional characteristics of the skin within the groups, as s of skin in patients and healthy individuals, obtained with the help of dermatoscopy on a digital video camera "Aramo $S G$ "

\begin{tabular}{|l|l|l|l|l|l|}
\hline Groups & \multicolumn{1}{|c|}{ Index } & \multicolumn{1}{|c|}{ Mean } & \multicolumn{1}{c|}{ Median } & \multicolumn{1}{c|}{ Min } & \multicolumn{1}{c|}{ Max } \\
\hline \multirow{2}{*}{} & $\mathrm{pH}$ & $7,1 \pm 0,8$ & 7,0 & 6,8 & 7,5 \\
\cline { 2 - 6 } & moisture & $32,7 \pm 7,8^{*}$ & 34,0 & 7,0 & 45,0 \\
\cline { 2 - 7 } & fat contentU & $18,2 \pm 18,5^{*}$ & 12,5 & 2,0 & 94,0 \\
\hline
\end{tabular}




\begin{tabular}{|c|c|c|c|c|c|}
\hline & fat content $\mathrm{T}$ & $38,3 \pm 25,9 *$ & 30,5 & 4,0 & 100,0 \\
\hline & pigmentation & $44,6 \pm 28,8 *$ & 40,0 & 9,0 & 100,0 \\
\hline & pore size & $32,4 \pm 15,9 *$ & 22,0 & 5,0 & 55,0 \\
\hline & elasticity & $59,5 \pm 16,2 *$ & 60,0 & 25,0 & 95,0 \\
\hline & smoothness & $46,8 \pm 9,0^{*}$ & 46,0 & 31,0 & 70,0 \\
\hline \multirow{8}{*}{$\begin{array}{l}\widehat{\theta} \\
\text { II } \\
\Xi \\
0 \\
0 \\
0 \\
0 \\
=\end{array}$} & $\mathrm{pH}$ & $6,0 \pm 0,9$ & 6,0 & 5,0 & 7,0 \\
\hline & moisture & $34,6 \pm 6,3^{*}$ & 35,0 & 15,0 & 50,0 \\
\hline & fat contentU & $15,4 \pm 18,3$ & 10,0 & 0 & 84,0 \\
\hline & fat content $\mathrm{T}$ & $30,0 \pm 21,0$ & 25,0 & 2,0 & 100,0 \\
\hline & pigmentation & $41,7 \pm 23,8$ & 38,0 & 5,0 & 100,0 \\
\hline & pore size & $19,0 \pm 17,4$ & 19,0 & 5,0 & 81,0 \\
\hline & elasticity & $63,7 \pm 16,3$ & 65,0 & 30,0 & 100,0 \\
\hline & smoothness & $45,2 \pm 8,4^{*}$ & 46,0 & 31,0 & 64,0 \\
\hline \multirow{8}{*}{$\begin{array}{l}\text { ठ̊ } \\
\text { II } \\
\Xi \\
0 \\
0 \\
0 \\
\Xi \\
\Xi\end{array}$} & $\mathrm{pH}$ & $5,5 \pm 0,7$ & 6,0 & 5,0 & 7,0 \\
\hline & moisture & $45,6 \pm 5,1^{*}$ & 45,0 & 33,0 & 50,0 \\
\hline & fat contentU & $8,8 \pm 10,4^{*}$ & 5,0 & 1,0 & 54,0 \\
\hline & fat content $\mathrm{T}$ & $22,9 \pm 16,5^{*}$ & 19,0 & 1,0 & 68,0 \\
\hline & pigmentation & $29,5 \pm 22,6^{*}$ & 24,0 & 1,0 & 87,0 \\
\hline & pore size & $9,7 \pm 3,4$ & 23,0 & 4,0 & 48,0 \\
\hline & elasticity & $69,3 \pm 13,9 *$ & 70,0 & 40,0 & 90,0 \\
\hline & smoothness & $38,7 \pm 7,0^{*}$ & 38,0 & 27,0 & 54,0 \\
\hline
\end{tabular}

(Kruskal - WallisTest; $\mathrm{p}<0,05$ )

There is an example of a dry skin obtained by the digital video camera"Aramo SG" (Figure 4).
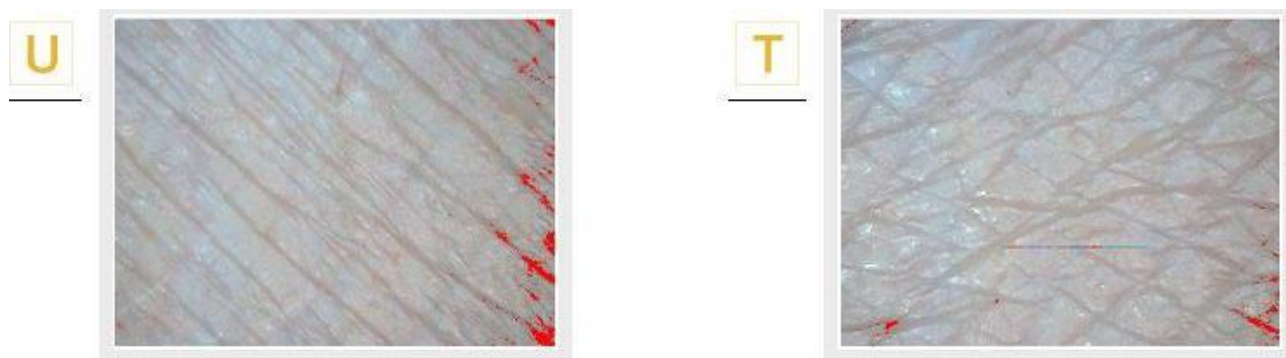

Fig4. Example of a dry skin. Photo was made by "Aramo SG" digital camera

Pictures 5 and 6 represent a smoothness and pigmentation respectively. Photo was made by the digital video camera "Aramo SG".
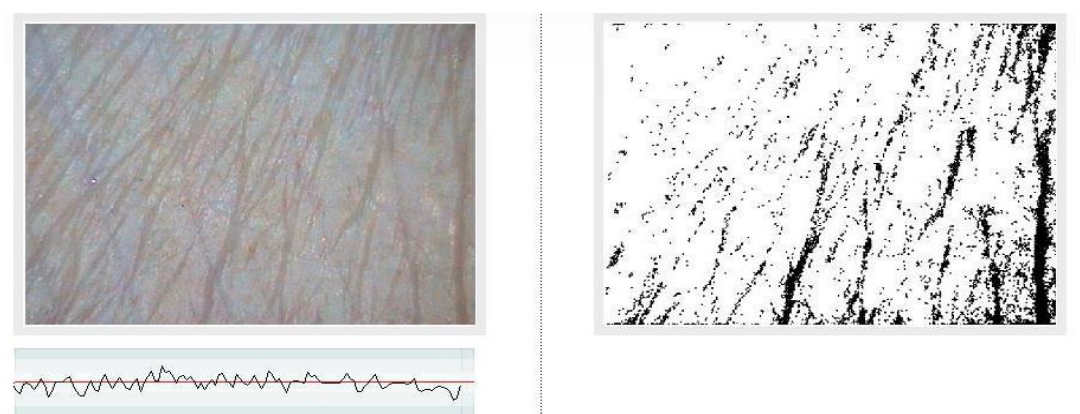

Fig5. Smoothness of the skin. (thedigital video camera"Aramo $\left.S G^{\prime \prime}\right)$.
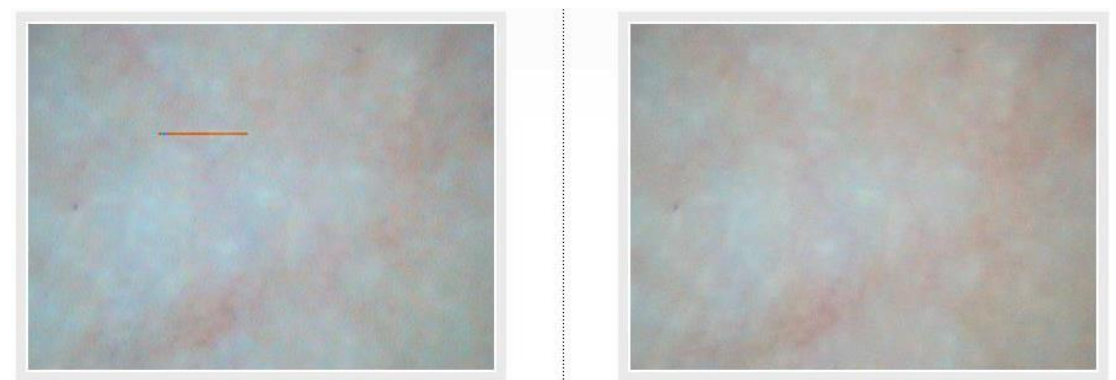

Fig6. Skin Pigmentation. (the digital video camera"Aramo $\left.S G^{\prime \prime}\right)$. 
According to the skin fatness levels in the $\mathrm{U}$ and $\mathrm{T}$ zones, the "Aramo SG" digital video camera automatically detects the type of skin (normal, dry, oily, combined). Table 2 shows the distribution of skin types among participants enrolled in the study groups.

Table2. Distribution of skin types in respondents

\begin{tabular}{|l|l|l|l|l|l|}
\hline & \multicolumn{1}{|c|}{ Normal $(\mathrm{n} ; \%)$} & Dry $(\mathrm{n} ; \%)$ & \multicolumn{1}{|c|}{ Oily $(\mathrm{n} ; \%)$} & \multicolumn{1}{|c|}{ Combined $(\mathrm{n} ; \%)$} & \multicolumn{1}{c|}{ Total $(\mathrm{n} ; \%)$} \\
\hline Igroup & $2 ; 1,1$ & $2 ; 1,1$ & $36 ; 20,0^{*}$ & $20 ; 11,1$ & $60 ; 33,3$ \\
\hline IIgroup & $8 ; 4,4$ & $12 ; 6,7$ & $34 ; 18,9^{*}$ & $6 ; 3,3$ & $60 ; 33,3$ \\
\hline IIIgroup & $50 ; 27,9^{*}$ & $2 ; 1,1$ & $2 ; 1,1$ & $6 ; 3,3$ & $60 ; 33,4$ \\
\hline Total & $60 ; 33,4$ & $16 ; 8,9$ & $72 ; 40,0$ & $32 ; 17,7$ & $180 ; 100$ \\
\hline
\end{tabular}

$*-\mathrm{p}<0,05$

Morfofunctional characteristics of the skin were also evaluated depending on the duration of the disease (Table 3).

Table3. Morphofunctional characteristics of the skin of patients with rosacea depending on the duration of the disease

\begin{tabular}{|c|c|c|c|c|c|c|}
\hline \multirow[t]{3}{*}{ Index } & \multicolumn{6}{|c|}{ Duration of the disease } \\
\hline & \multicolumn{3}{|c|}{ Igroup } & \multicolumn{3}{|c|}{ IIgroup } \\
\hline & $<1$ year & $1-5$ years & $6-10$ years & $<1$ year & $1-5$ years & 5-10 years \\
\hline $\mathrm{pH}$ & $7,2 \pm 0,3$ & $7,4 \pm 0,43$ & $7,5 \pm 0,82$ & $5,7 \pm 0,7$ & $6,2 \pm 0,7$ & $7,0 \pm 0,9$ \\
\hline moisture & $33 \pm 5,93$ & $32,7 \pm 5,97$ & $32,7 \pm 7,84$ & $37 \pm 2,6$ & $35 \pm 3,9$ & $34,5 \pm 4,2$ \\
\hline fat contentU & $13,7 \pm 7,9$ & $15,4 \pm 10,4$ & $18,2 \pm 18,7$ & $14,7 \pm 11,7$ & $15,3 \pm 14,3$ & $15,3 \pm 18,0$ \\
\hline fat content $\mathrm{T}$ & $36,3 \pm 18,4$ & $38,0 \pm 21,3$ & $38,25 \pm 26,1$ & $27,5 \pm 16,8$ & $27,3 \pm 17,6$ & $27,5 \pm 21,7$ \\
\hline pigmentation & $37,3 \pm 20,0$ & $40,1 \pm 24,3$ & $44,6 \pm 29,07$ & $36,8 \pm 16,4$ & $42,0 \pm 15,9$ & $41,7 \pm 23,8$ \\
\hline pore size & $25,5 \pm 14,3$ & $25,9 \pm 15,8$ & $26,4 \pm 16,0$ & $19,8 \pm 11,9$ & $24,0 \pm 13,2$ & $24,0 \pm 17,4$ \\
\hline elasticity & $64,0 \pm 13,9$ & $60,2 \pm 14,1$ & $59,5 \pm 16,3$ & $65,8 \pm 12,3$ & $62,4 \pm 14,8$ & $63,7 \pm 16,3$ \\
\hline smoothness & $46,0 \pm 7,8$ & $46,0 \pm 8,7$ & $46,8 \pm 9,1$ & $41,8 \pm 5,9$ & $42,0 \pm 6,3$ & $45,2 \pm 8,4$ \\
\hline
\end{tabular}

\section{DISCUSSION}

A comparative analysis of the morph functional characteristics of the facial skin of group I and II patients and healthy individuals (group III) revealed statistically significant differences in moisture index (between groups I and III and groups II and III), fat content (between groups I, II and III), pigmentation (between groups I and III), elasticity (between groups I and III), smoothness (between groups I and III and groups II and III), pore size (between groups I, II and III).

The statistical difference in humidity between groups is associated with increased trans epidermal loss of moisture in patients with rosacea compared with healthy individuals.

Increased skin fatness in patients with rosacea in the $\mathrm{U}$ and $\mathrm{T}$ zones of the face is associated with increased production of sebum at rosacea.

A statistically significant difference in the elasticity and smoothness of the skin is revealed between the three groups and is associated with the presence of inflammatory and noninflammatory primary and secondary elements on the skin of the face that alter the normal architectonics of the skin.

The increase in the size of the excretory ducts of the sebaceous glands is associated with the presence of rosacea, increased production of sebaceous glands of sebum, a violation of its outflow and an increase in the size of the sebaceous glands, which creates favourable conditions not only for the formation of diseases, but also for successful colonization by Demodexmites.

The shift of the $\mathrm{pH}$ level in patients of the group I to the alkaline side indicates a decrease in the protective properties of the skin, which is likely to be a favourable condition for infection by Demodexmites.

Analysing the types of skin in the respondents of the three groups, it was found that in the I and II groups the patients were statistically significantly more likely to have oily skin $(\mathrm{n}=$ $36,20.0 \%$ and $\mathrm{n}=34,18.9 \%)(\mathrm{p}<0,05)$. In healthy individuals of group III, normal skin (n $=50,27.9 \%)(\mathrm{p}<0,05)$ was statistically significantly more likely to predominate.

Thus, in patients with rosacea, the facial skin is oilier than in healthy individuals. According to the data obtained, it was found that in the presence of a tick in patients there is a statistically significant change in the functional characteristics of the skin.

Although no significant differences in the results were found, but as can be seen from 
Table 3, the morphofunctional characteristics of the skin gradually change toward deterioration, depending on the presence of Demodex mites and the duration of the disease.

\section{CONCLUSION}

When comparing the morphofunctional characteristics of the facial skin obtained on a digital video camera "Aramo SG", statistical differences between the groups were revealed. In the presence of a mites, the patients have statistically significant changes in the morphofunctional characteristics of the skin in the direction of deterioration in moisture, fat content, pigmentation, pore size, elasticity, skin smoothness and $\mathrm{pH}$ shift to alkaline side compared to patients not infected with mites and healthy $(\mathrm{p}<0,05)$. During the observation, it was noted that the parameters of the morpho functional characteristics of the skin become worse with increasing duration of the disease.

\section{REFERENCES}

[1] Baima B., Sticherling M. Demodicidosis revisited. Acta DermVenereol. 2002; 82: 3-6

[2] Rufli T, Mumcuoglu Y. The hair follicle mites Demodex folliculorum and Demodex brevis: Biology and medical importance. A review. Dermatologica. 1981; 162:1-11

[3] Aylesworth R, Vance C. Demodex folliculorum and Demodex brevis in cutaneous biopsies. $\mathrm{J}$ Am AcadDermatol. 1982;7:583-9

[4] Basta- Juzbasic A, Subic JS, Ljubojevic S. Demodex folliculorum in development of dermatitis rosacei formissteroidica and rosacearelated diseases. Clin Dermatol. 2002;20:135-4

Citation: Kubanov Alexey, GallyamovaYulia, KravchenkoAnzhela, Characteristics of Facial Skin and Demodex Mites. ARC Journal of Dermatology. 2018; 3(2):12-16. doi:dx.doi.org/10.20431/2456-0022.0302004.

Copyright: (C) 2018 Authors. This is an open-access article distributed under the terms of the Creative Commons Attribution License, which permits unrestricted use, distribution, and reproduction in any medium, provided the original author and source are credited. 